\title{
A METHOD FOR THE DETECTION AND DETERMINATION OF ARSENIC AND ANTIMONY IN THE PRES- ENCE OF ORGANIC MATTER.
}

By F. A. Norton AND A. E. Koch.

Received July 28, 1905 .

THE volatility of these metals precludes the incineration of organic matter containing them for their detection or estimation as is so conveniently done with most metals. A number of methods such as those of Fresenitus and Babo, of Gautier, and of Danger and Flandin have been proposed, which have in view the destruction of the organic matter as completely as possible without material loss of the antimony or arsenic. All of these methods, however, fall more or less short of complete destruction of the organic matter and render subsequent determinations difficult or less satisfactory.

It occurred to us while working upon arsenic determinations in the presence of organic matter, that moist combustion with sulphuric acid might be employed to destroy the organic matter and permit of ready detection or estimation of either arsenic or antimony. This method was suggested by Pattinson's method ${ }^{1}$ for the decomposition of arsenious sulphide with strong sulphuric acid and titration of the resulting arsenious acid with standard iodine solution for the estimation of arsenic. Sutton, ${ }^{2}$ speaking of this method, says: "Experiments show that there is no loss of arsenious acid by volatilization when arsenious sulphide is decomposed by heating with strong sulphuric acid in the manner described." Various determinations by the authors of this paper show quite conclusively that compounds of either arsenic or antimony are not volatilized on digestion with sulphuric acid, with the exception of arsenic trichloride, and it is but slightly volatilized, if at all, when much organic matter is present. This being the case, tissues or organic matter containing arsenic or antimony, if the metal is present in sufficient quantity or if there is not too much organic matter to oxidize, may be introduced directly into a Kjeldah1 digestion flask or suitable retort, and digested with concentrated sulphuric acid until clear or of a straw color. If but little arsenic or antimony is present, it would be impracticable to subject the necessary amount of the substance

$1 \mathrm{~J}$. Soc. Chem, Ind., 17, 2 I I (1898).

2 Sutton's "Volumetric Analysis," Ninth Edition, p. 159. 
to moist combustion, and some method of extraction should be employed and the extract digested with the sulphuric acid.

For arsenic the favorite method with toxicologists where quantitative determinations are not to be made, is to digest the tissues with one-sixth hydrochloric acid for an hour at gentle boiling. A number of determinations made by us with different solvents for rapid extraction gave the best results with dilute hydrochloric acid as above. The extract obtained by straining and washing the tissues after extraction, we found could be readily digested with concentrated sulphuric acid without material loss of arsenic. Approximately 80 per cent. of the arsenic present was readily extracted by this method on each of several trials. With antimony we found that approximately 90 per cent. of the antimony as antimony and potassium tartrate could be extracted from organic matter by treatment with dilute tartaric acid instead of the hydrochloric acid, and digestion of the extract with sulphuric acid.

When it is desired to recover all of the arsenic or antimony present, the tissues, if in too large quantity to digest directly, should first be treated according to one of the more complex methods of extraction. We have found the modification of the Gautier method, as given by Peterson and Haines, ${ }^{1}$ to give the best results for arsenic, as there is no danger of volatilization of arsenic as trichloride, hydrochloric acid not being used. With antimony, treatment of the tissues according to the method of Fresenius and Babo gives satisfactory results and is more rapid than the Gautier method.

With either metal, the first step is to obtain the arsenic or antimony free from organic matter as above with as small an excess of sulphuric acid as possible. Usually $20 \mathrm{cc}$. of sulphuric acid (sp. gr. 1.84) is sufficient to oxidize 5 grams of tissue or the extract obtained from 100 grams. If qualitative tests are to be made, the sulphuric acid residue may be diluted, and aliquot portions taken for the different tests. For the Marsh test, the addition of arsenic-free zinc in a suitable apparatus is all that is necessary, unless the acid solution has been rendered too dilute. For precipitation as sulphide or application of the Reinsch test the acid solution should be neutralized with soda and then acidified with hydrochloric acid.

1 Peterson and Haines' "Text-book of Legal Medicine and Toxicology," Vol. II, p. 324.. 


\section{QUANTITATIVE DETERMINATION.}

This may be readily accomplished volumetrically by Mohr's method. The arsenic, no matter in what condition it may have been present, is reduced by the sulphur dioxide liberated in the digestion to arsenious acid, nitrates are decomposed and salts present reduced to sulphates. Thus in general no interfering bodies are left in the acid residue and after neutralization the arsenious acid can be titrated with standard iodine solution. Antimony, in the digestion with sulphuric acid, is converted into subsulphate, and on neutralization of the acid residue with potash in slight excess and acidification with tartaric acid, is converted into antimony and potassium tartrate which may be titrated with standard iodine solution, after addition of sodium bicarbonate solution.

The following determinations by the authors will show the applicability and accuracy of the method:

With Arsenic.-Four different portions of to grams of meat and $2.5 \mathrm{mg}$. of arsenious acid were introduced into $\mathrm{Kjeldahl}$ digestion flasks and digested with pure sulphuric acid until colorless or of a straw color. The flasks were then cooled, the residues diluted, nearly neutralized with soda free from oxidizable substances, and a sufficient excess of a cold saturated solution of sodium bicarbonate added, then titrated with tenth-normal iodine solution. Results follow:

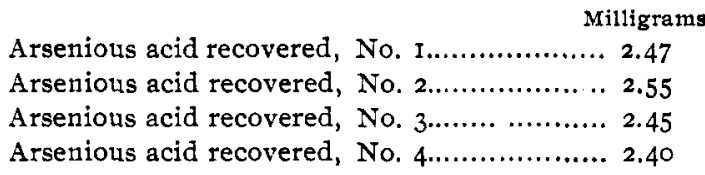

In the second trial, two portions of Ioo grams of finely divided meat containing $50 \mathrm{mg}$. each of arsenic trioxide were extracted by the following modification of the Gautier method as given by Peterson and Haines: To the meat in a porcelain capsule of about $600 \mathrm{cc}$. capacity were added 50 grams of strong pure nitric acid and I gram of pure sulphuric acid, and the mixture heated gently, at first, until liquefaction took place and the material became thick. It was then removed from the heat and ro grams of pure sulphuric acid added, then heated again, care being taken to prevent carbonization. A little nitric acid was added from time to time until on heating it to a point where heavy vapors of sul- 
phuric acid were given off, there was left in the capsule a brown liquid practically uncarbonizable at the temperature at which sulphuric acid begins to boil. When no further oxidizing effect was observed, the nitric acid was driven off by heat, the material allowed to cool, a little sulphuric acid added and the small quantity of residual brown liquid poured with constant stirring into $600 \mathrm{cc}$. of cold distilled water. The liquid was then filtered and the filtrate and washings made up to $1000 \mathrm{cc}$. Duplicate aliquot portions of the extracts representing $\mathrm{i} 2.5 \mathrm{mg}$. of arsenious acid were then transferred to Kjeldahl digestion flasks, $20 \mathrm{cc}$. of sulphuric acid added, and the liquid digested until nearly clear, then neutralized and titrated as above. Results follow:

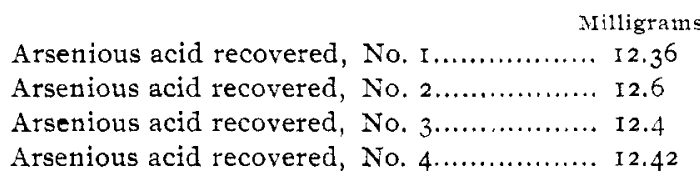

With Antimony.-Two portions of Ioo grams of finely divided meat, each containing $200 \mathrm{mg}$. of pure recrystallized antimony and potassium tartrate, were treated, according to Fresenius and Babo, as follows: The meat in a porcelain capsule on a water-bath was brought to the consistency of thin gruel with distilled water, and roo grams of pure hydrochloric acid added, then small quantities of potassium chlorate were added from time to time until the solid matters disappeared and the liquid became a clear yellow. The heat was then continued until the odor of chlorine disappeared, when the liquid was strained and the filtrate and washings made up to $250 \mathrm{cc}$. Aliquot portions of each extract representing $20 \mathrm{mg}$. of antimony and potassium tartrate were then digested with sulphuric acid, the acid residue diluted, neutralized with slight excess of potassium hydroxide, the solution slightly acidified with tartaric acid, a sufficient quantity of solution of sodium bicarbonate added and immediately titrated with tenthnormal iodine solution. Results follow:

\footnotetext{
Milligrams.

Antimony and potassium tartrate recovered, No. I......... 19.9

Antimony and potassium tartrate recovered, No. 2....... 19.87

Antimony and potassium tartrate recovered, No. 3........ I 9.87

Antimony and potassium tartrate recovered, No. 4....... 19.95
}

Gravimetric methods of estimation might be desirable in some instances, though if care is taken to establish the absence of interfering substances, pure chemicals are used and the solutions are 
standardized under conditions of titration, the above process would be as reliable as any volumetric determination and gives a comparatively rapid and easy method of estimation, as the moist combustion takes only from three to six hours and the titration but a few minutes. If desired, antimony or arsenic can be confirmed in the residue from the titration by either the Reinsch or Marsh tests.

Gravimetric methods may also be applied to the acid residue from the digestion of tissues or their extracts. Gravimetric results, however, obtained by us were not as satisfactory as the volumetric. Determinations of arsenic as sulphide, and antimony both as sulphide and tetroxide, after neutralization upon acid residues obtained as for the volumetric determinations, gave somewhat high results, even when the respective sulphides were dissolved with ammonia and ammonium sulphide and reprecipitated to free from contaminating salts. The estimation of arsenic as mirror, according to the method of Fresenius, ${ }^{1}$ on the acid residue, worked very nicely, there being no frothing as in the case of employing the Gautier extract as reported by Fresenius. The results, however, were somewhat low on account of the difficulty in decomposing all of the hydrogen arsenide during its passage through the heated tube. The delicacy of this method is shown by the following experiment: A rat weighing about 200 grams was poisoned with arsenic and its liver digested with sulphuric acid until the acid residue was clear. Arsenic was then determined according to Fresenius, a distinct arsenical mirror weighing half a milligram being obtained.

\section{SUMMARY.}

Moist combustion can readily be employed to free arsenic or antimony in tissues or extracts from interfering organic matter for qualitative tests. The acid residues from the digestion of extracts or tissues can be immediately titrated, after neutralization, with standard iodine solution for the quantitative estimation of arsenic or antimony.

The acid residue from the digestion of tissues or extracts may also be employed for gravimetric determinations, or with arsenic for estimation as mirror.

ChEMICAI IABORATORY, SOUTh DAKOTA

EXPERIMENT STATION.

1 Fresenius" "Quantitative Chemical Analysis," Sixth German Edition, by Cohn, Vol. II, p. 693. 